\title{
An algorithm for train rescheduling using rescheduling pattern description language $\mathbf{R}$
}

\author{
C. Hirai ${ }^{1}$, N. Tomii ${ }^{1}$, Y. Tashiro ${ }^{2}$, S. Kondou ${ }^{1} \&$ A. Fujimori ${ }^{3}$ \\ ${ }^{1}$ Transport Information Technology Division, \\ Railway Technical Research Institute, Japan \\ ${ }^{2}$ Kyushu Railway Company, Japan \\ ${ }^{3}$ Hokkaido Railway Company, Japan
}

\begin{abstract}
We propose an algorithm for automatic train rescheduling with a train rescheduling pattern language processing system. Intended for restoration from heavy train traffic disruption, our proposed algorithm has inherent abilities to make effective train rescheduling plans. While the previous algorithm tries to make a train rescheduling plan in small steps, the proposed one surveys the train timetable at first and applies "train rescheduling patterns" to prepare rescheduling plans. Applying actual train schedule data, we have confirmed that our algorithm works satisfactorily. For severe train traffic disruption caused by an accident, in particular, requiring the suspension of train operations for more than an hour, the algorithm is helpful for preparation of adequate rescheduling plans for practical application.

Keywords: train rescheduling, pattern description, train rescheduling pattern, train traffic disruption, train traffic control, framework of train rescheduling system.
\end{abstract}

\section{Introduction}

Train traffic is sometimes disrupted when accidents, natural disasters or technical problems occur on railway lines. In order to restore disrupted services, railways have continuously made a series of modifications to the current train schedules. Such a task is termed as train rescheduling [1,2]. It is quite important for railway companies to prepare an adequate train rescheduling plan hereinafter referred to "the plan" whenever train traffic disrupted. 
At present, expert train dispatchers are involved in train rescheduling. They constantly monitor daily train operations closely at their post. Once an accident that likely cause disruption of transport services occurs, train dispatchers collect information about it and prepare "the plan" based on their previous experiences and intuition. They take into account not only the future train traffic and the passenger density but also unforeseen problems.

It is required to introduce an algorithm for automatic train rescheduling. In order to maintain stable transport services, railway companies require a mechanism which ensures the quality of "the plan". Recently computer systems adopted in operational services to assist train dispatchers in charge of train rescheduling, refer to $[3,4]$. The systems, however, have a lack in a certain functions required to formulate "the plans" automatically.

In this paper, we propose an algorithm for automatic train rescheduling. Our algorithm based on a technique that applies experts' knowledge as "train rescheduling patterns" hereinafter referred to "the patterns" to automatic computation of "the plan". Some railway companies recognize specifiable parts of the experts' knowledge as "the patterns" to restore disrupted train traffic effectively. In order to build a practical algorithm for automatic train rescheduling, a use of "the patterns" is recommendable.

On the other hand, we have to consider the fact that a mere use of "the patterns" is insufficient to prepare effective and practical plans. Due to uncertainty of occurrences of accidents, it is difficult to prepare complete train rescheduling patterns to suit to every situation. Such circumstance results in a need of a mechanism that modifies "the plan" prepared by only application of "the patterns".

For the sake of constructing an automatic train rescheduling algorithm, we have to solve the following problems:

1. Establish a language to describe "the patterns".

2. Develop a language processing system (an interpreter) to apply "the patterns".

3. Construct a framework of an automatic train rescheduling system to modify the plan created by the interpreter.

We have solved above three problems, and implemented an algorithm for automatic train rescheduling. Applying actual train schedule data, we have confirmed that our algorithm works are satisfactory.

\section{Train rescheduling pattern}

\subsection{Train rescheduling pattern}

Some railway companies recognize specifiable parts of the experts' knowledge as "the patterns" to restore effectively disrupted train traffic. Each train dispatcher has his own strategy in preparation of "the plan", which causes variations in "the plans". For example, under the same conditions, a dispatcher adopts a plan by which disrupted traffic restorable as immediately as possible while another dispatcher selects a plan that maintains sufficient traffic capacity. 
In order to provide stable and punctual transportation services to public, every train dispatcher needs to own common and compatible strategies for train rescheduling. Railway companies accordingly have taken a step under which every train dispatcher utilizes "the patterns".

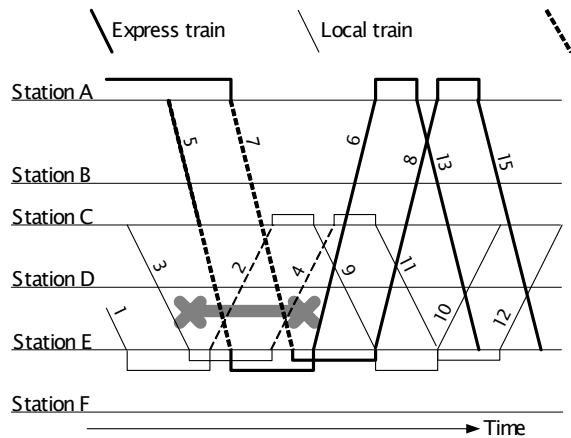

(a) Initial schedule

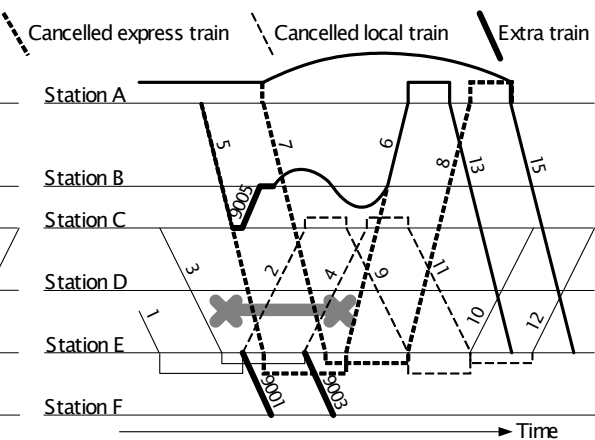

(b) Resultant schedule

Figure 1: Example of train rescheduling.

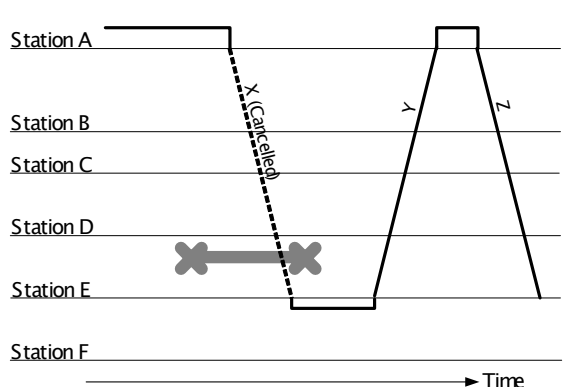

(a) Initial schedule (Train $X$ is cancelled)

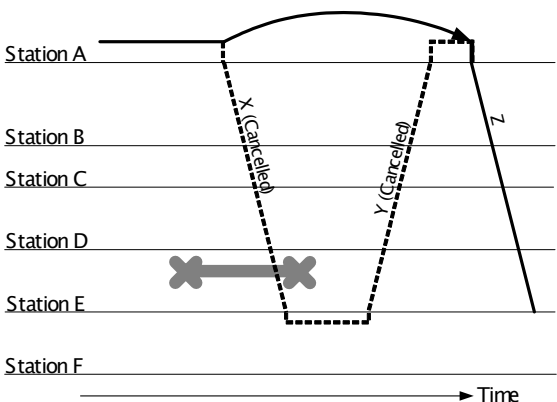

(b) Resultant schedule

Figure 2: Train rescheduling pattern 1 (TRP-1).

\subsection{Examples of applying train rescheduling patterns}

Figure 1 indicates examples how "the patterns" are applicable when train traffic is disrupted. Figure 1(a) shows the initial schedule while Figure 1(b) shows the resultant schedule. A thick diagonal line indicates an express train, and a thin diagonal line shows a local train. Express trains operated between station-A and station-E; and local trains between station-C and station-E.

We assume that station-B and station-F have sufficient tracks to accommodate temporary train-sets. On the contrary, there is no extra track available at both station-C and station-E. Grey thick line indicates a location and an affected time interval of the accident where trains are inoperable. A broken diagonal line indicates a cancellation of the corresponding train.

Figure 1(a) illustrates initial schedule that includes train cancellations immediately after the accident. We assume that trains encountered with the 
accident will be likely cancelled as initial operations. In Figure 1(a), the initially cancelled trains are train-5, train-7, train-2 and train-4.

Figure 1(b) shows a resultant schedule after applying the following patterns.

Figure 2 shows a train rescheduling pattern 1 (TRP-1). In case an express train- $\mathrm{X}$ is cancelled between station- $\mathrm{A}$ and station- $\mathrm{E}$, the next express train- $\mathrm{Y}$ from station-E cancelled. A train-set for an express train-X waits at station-A, and operated as the next express train-Z.

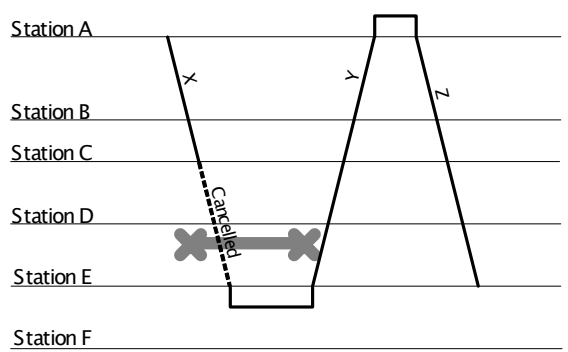

(a) Initial schedule (Train X is partially cancelled)

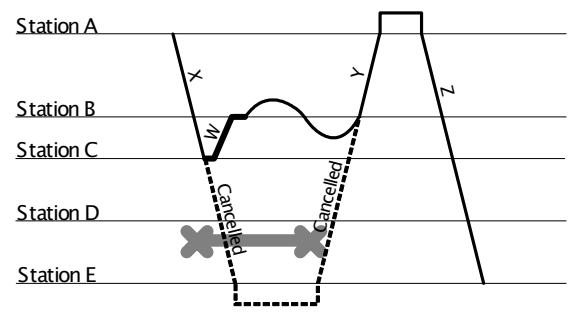

Station F

(b) Resultant schedule

Figure 3: Train rescheduling pattern 2 (TRP-2).

Figure 3 shows a train rescheduling pattern 2 (TRP-2). In case an express train- $X$ cancelled between station- $C$ and station- $E$, the next express train- $Y$ cancelled between station-E and station-B. An extra train-W is set from station-C to station-B. A train-set for train-X operated as a train-W and waits at station-B. After that, the train-set operated as train-Y from station-B to station-A.

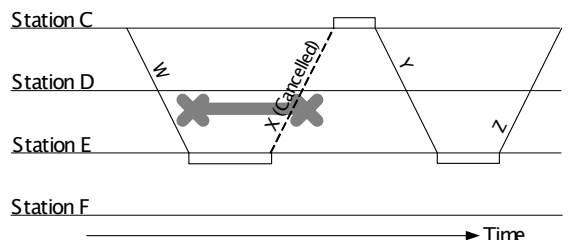

(a) Initial schedule (Train $\mathrm{X}$ is cancelled)

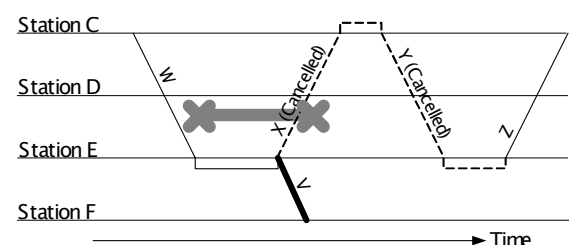

(b) Resultant schedule

Figure 4: Train rescheduling pattern 3 (TRP-3).

Figure 4 shows a train rescheduling pattern 3 (TRP-3). In case a local train-X cancelled between station-E and station- $\mathrm{C}$, the next local train- $\mathrm{Y}$ cancelled between station-C and station-E. An extra train- $\mathrm{V}$ is set from station- $\mathrm{E}$ to station$\mathrm{F}$. A train-set for train-X waits at station-F until the next operation is decided.

A resultant schedule in Figure 1(b) is derived from application of TRP-1, TRP-2 and TRP-3 to the corresponding cancellations respectively as shown in Figure 1(a). 


\subsection{Background of introducing train rescheduling patterns}

Based on our hearing investigation of several railway companies, we can summarize reasons to make use of "the patterns" as follows:

a. Provide stable transport services. It is possible to reduce variations due to different strategies taken by each train dispatcher in preparation train rescheduling tasks.

b. Perform train rescheduling task smoothly. Train rescheduling operations involve many people; train dispatchers, drivers, conductors, station staff, etc. It is necessary for them to coordinate closely each other to avoid any failure. Familiarized with the detail of the plan in advance, every staff involved in the work is capable of fulfilling their activities with confidence.

c. Avoid unforeseen problems. Skilled train dispatcher has expertise to avoid unpredictable trouble. Though it is not possible for them to explicitly explain the details of the trouble, in many occasions there are covert information not disclosed to the public. Hence we can incorporate the expertise into "the patterns" beforehand; train dispatchers are able to take advantage of expertise of other dispatchers.

d. Realize train rescheduling operations that reflect a company policy of railways. It is possible to incorporate the policy into "the patterns" beforehand.

Considering the concept of railway companies mentioned as above, we conclude that a practical algorithm for automatic train rescheduling should utilize "the patterns".

In addition, the utilization of "the patterns" creates a reduction of computing time. Our approach includes a simplified procedure; merely applying "the patterns" to the current schedule. In comparison with an algorithm that enables to prepare "the plans" successively from the beginning, our approach enables to construct an algorithm by which "the plans" are prepared within a short period.

\subsection{Problems to be solved to introduce train rescheduling patterns}

For the sake of constructing an automatic train rescheduling algorithm, we have to solve the following problems:

1. Establish a language to describe train rescheduling patterns: Up to now, "the patterns" are drawn in a natural language or drawn graphically as shown in Figure 2, 3 and 4. Therefore, it is necessary to establish a language that enables to represent them in a computer-recognizable form.

2. Develop a language processing system (an interpreter) to apply "the patterns": A mechanism applying "the patterns" to the current train schedule is essential. In other words, the following functions are required.

- A function to find a location where "the patterns" is applicable to the current schedule: in Figure 2, cancelled train-X should be found to apply TRP-1. 
- A function to assign train names of the current train schedule to "the pattern": in Figure 1(b) and Figure 4, it is necessary to assign train-2 and train-4 to train-X.

- A function to output operation instructions: In Figure 1(b), the output should be cancellations of train- 6 , train- 8 , train- 9 and train-11, settings of train-9005, train-9001 and train-9003, and related train connections.

3. Construct a framework of an automatic train rescheduling system to modify the plan created by the interpreter: The only use of "the patterns" is insufficient to prepare a practical rescheduling plan. It is difficult to prepare "the patterns" for every single case. Not all trains are applicable; therefore, a framework that modifies the results of applying "the patterns" is required.

We have settled these problems. For each problem, our approach is as follows:

1. Introduce a pattern description language named " $R$ ".

2. Develop a pattern description language processing system named "R-interpreter".

3. Construct a framework for comprehensive train rescheduling system.

\section{Pattern description language $\mathbf{R}$ and $\mathrm{R}$-interpreter}

We introduce a language named " $R$ " to describe "the patterns". In addition, we have implemented a language processing system named "R-interpreter" to interpret descriptive contents written in R.

We designate "the patterns" written in R "R-rule." R-rule describes actions that executed when an event occurs. An event corresponds to a change of train schedule like a train cancellation.

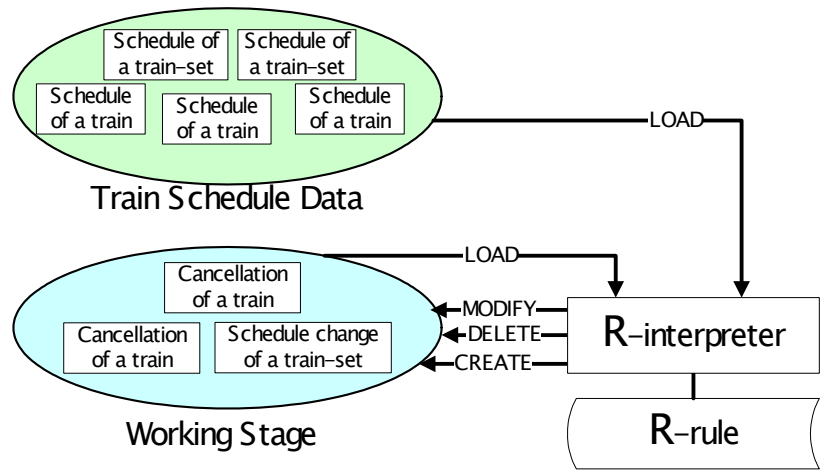

Figure 5: Operations of R-interpreter.

Figure 5 illustrates operations of R-interpreter. R-interpreter handles events at Working Stage based on R-rule, train schedule data and the current states of Working Stage. When R-interpreter is not able to find any of R-rule applicable to the current state, R-interpreter aborts its operations. 
R-rule consists of three parts as below:

(1) (for_each) section

Describe an event that behaves a trigger of "the pattern". R-interpreter searches the initial schedule for the trigger. In case that the trigger is found, R-interpreter try to check the conditions described in (if_exists) section.

(2) (if_exists) section

Describe conditions for actions.

(3) (then) section

Describe contents of actions.

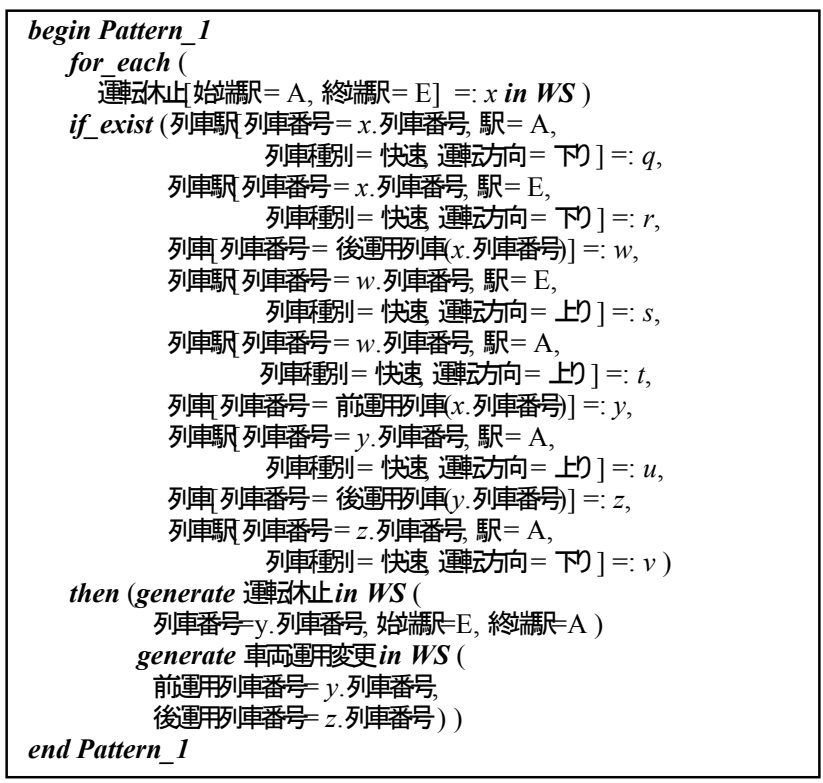

Figure 6: Description of TRP-1 in train rescheduling pattern language R.

Figure 6 shows an example that describes TRP-1 as shown in Figure 2. This description includes Japanese characters (Kanji and Kana characters), readily usable for Japanese train dispatchers. It is obviously possible to modify R-interpreter to deal with R-rule specified with only alphabetical characters.

The descriptive content in the (for_each) section in Figure 6 represents a cancellation of a train between station-A and station-E. This cancellation corresponds to that of train-X in Figure 2.

The description in the (if_exist) section represents conditions to be checked. In the (if_exist) section in Figure 6, the following conditions are specified;

- Existence of express train-Y that is operated from station-E to station-A (the same operating section as train-X's but the opposite direction).

- Existence of express train-Z that departs from station-A. 
The (then) section in Figure 6 specifies actions of TRP-1. These actions correspond to the resultant schedule in Figure 2 are as follows:

- Cancellation of train-Y

- A train-set for train- $\mathrm{X}$ is operated as train- $\mathrm{Z}$

\section{Framework of Train rescheduling system}

\subsection{Framework of train rescheduling system}

Train rescheduling is a complicated and large-scale task. A train rescheduling system, which supports train rescheduling tasks, should have a comprehensive structure.

Figure 7 indicates a framework for a comprehensive train rescheduling system. The framework consists of five subsystems: Train scheduling subsystem, Train-set rescheduling subsystem, Train rescheduling (in the narrow sense) subsystem, Crew rescheduling subsystem and Shunting rescheduling subsystem. Each of the subsystems exchanges relevant information and prepares each scheduling plan in cooperation with other subsystems.

It is necessary to distinguish operations that have different reasons. For example, a reason for a cancellation of a train is likely to be one of the following:

a) In case where a train is express-service, a long delay loses its worth to arrive at a destiniation ealier.

b) There is no train-set that can be assigned to the train.

c) In order to restore the train traffic.

Therefore, Train scheduling subsystem, Train-set rescheduling subsystem, Train rescheduling (in the narrow sense) subsystem have each cancellation operation.

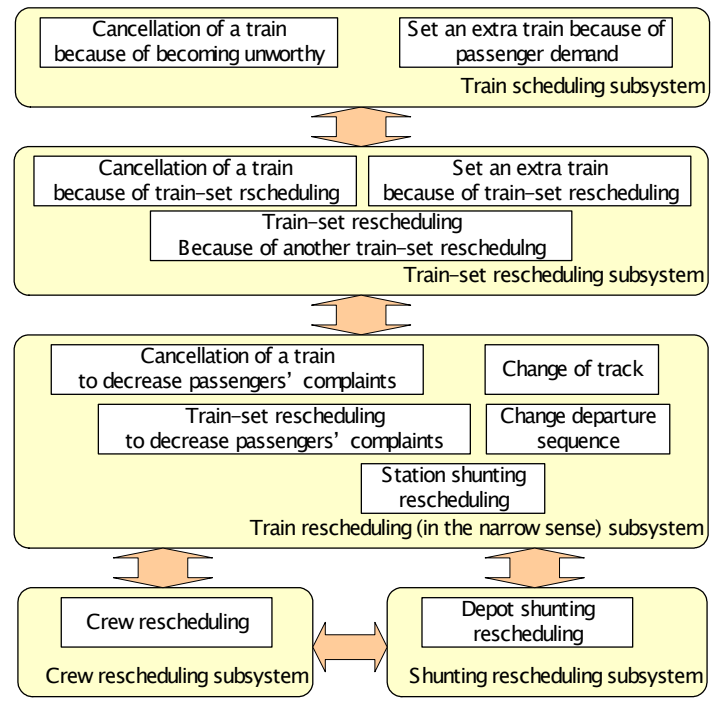

Figure 7: Framework of train-rescheduling system. 


\subsection{Train rescheduling algorithm}

We propose an algorithm based on the framework shown in Figure 7. With the example of Figure 1, operations of each subsystem are indicated as follows:

\subsubsection{Train scheduling subsystem}

Train scheduling subsystem determines cancellations of trains and creation of extra trains. In Figure 1(b), the following cancellations are determined based on usage of R-interpreter as shown in Section 2.

- $\quad$ Between station-C and station-E, train-2, train-4, train-5, train-9 and train-11 are cancelled.

- Between station-B and station-E, train-6 is cancelled.

- $\quad$ Between station-A and station-E, train-7 and train-8 are cancelled.

- Train-9001, train-9003 and train-9005 are created.

\subsubsection{Train-set rescheduling subsystem}

Train-set rescheduling subsystem prepares a train-set operating rescheduling plan. In Figure 1(b), no train-set is assigned to train-10 and train-12. Then, train-sets for train-9001 and train-9003 assigned to train-10 and train-12 respectively. A train-set rescheduling algorithm [5] is applicable in this instance.

\subsubsection{Train rescheduling (in the narrow sense) subsystem}

Employed a train reschedule algorithm [6], this subsystem solves the detailed problems like track conflicts. The following rescheduling operations are dealt with:

Cancellation, Extra trains, Change of track, Change departure sequence, Change train-set operating schedule and etc.

\subsubsection{Crew rescheduling subsystem}

For the rescheduling plan, this subsystem enables to prepare a crew rescheduling plan with a crew rescheduling algorithm [7].

\section{Results of experiments and evaluation of our algorithm}

We have evaluated effectiveness of our train rescheduling algorithm using actual train schedule data. For experiments, we selected a line that has 20 trains for an hour. An accident that requires an hour train suspension assumed as indicated by a black thick line, therefore, trains heading for the downward direction are not able to go into the location for an hour.

Table 1: Evaluation values of algorithms.

\begin{tabular}{c|c} 
Algorithm & Evaluation value \\
\hline \hline Without rescheduling (Figure 8(a)) & 2138 \\
\hline Existing algorithm [6] & 686 \\
\hline Proposed algorithm (Figure 8(b)) & 331
\end{tabular}


As an index for evaluation, we employed "dissatisfaction index" proposed in [6]. This index indicates the level of passengers' dissatisfaction, if the value is small; we regard the algorithm works satisfactory.

Table 1 indicates the result of the comparative evaluation. Because the value of the proposed algorithm is the smallest, we conclude that the proposed algorithm have made a better rescheduling plan than the existing algorithm. While the existing algorithm tries to prepare a train rescheduling plan tardily from the beginning, the proposed algorithm can utilize appropriate rescheduling policies given as R-rule in advance. Since R-rule contains expertise of skilful dispatcher, the proposed algorithm can prepare a practical rescheduling plan.

In addition, we can find obvious differences between disrupted schedule (no operation is executed) shown in Figure 8(a) and the resultant schedule shown in Figure 8(b).

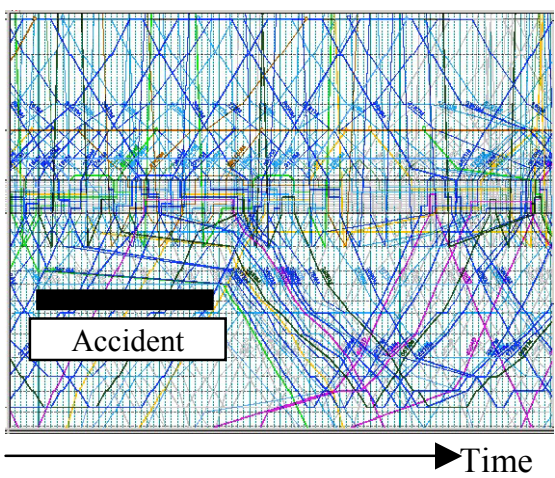

(a)

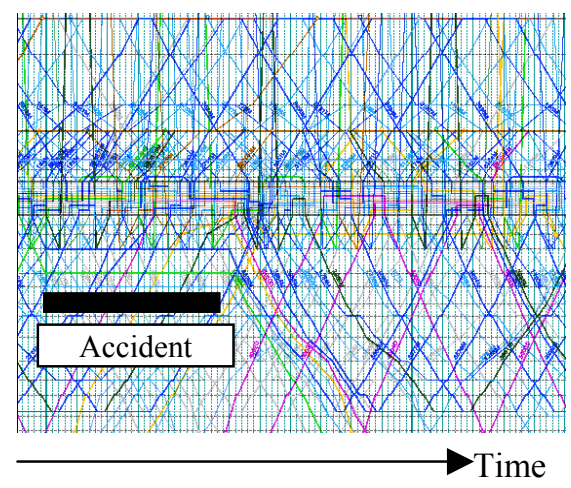

(b)

Figure 8: (a) Disrupted schedule and (b) Resultant schedule.

\section{Conclusions}

We proposed an algorithm for automatic train rescheduling with a train rescheduling pattern language processing system. Applying actual train schedule data, we have confirmed that our algorithm works satisfactory. Especially for heavy train traffic disruption, caused by an accident requiring a train suspension for more than an hour, the algorithm can produce a rescheduling plan suitable for practical use.

\section{References}

[1] Tomii, N., An Introduction to Railway Systems (in Japanese), Kyoritsu Publishing, pp. 64-78, 2001.

[2] Tomii, N., Techniques to make train diagrams for punctuality (in Japanese), Seizando Publishing, pp. 124-173, 2005. 
[3] Goodman, C. J. \& Takagi, R., Dynamic re-scheduling of trains after disruption, Computers in Railways IX, eds. J. Allan, C. A. Brebbia, R. J. Hill, G. Sciutto \& S. Sone, WIT Press, pp. 765-774, 2004.

[4] Nakamura, T. \& Ihara, K., The present situation and problems of train traffic control systems (in Japanese), IEEJ Journal, 124(5), pp.279-283, 2004.

[5] Hirai, C. Tomii, N. \& Tanabe, N., An algorithm for train set scheduling problems toward Cyber-Rail (in Japanese), Transportation and Electric Railway, TER-02-46, IEEJ, 2002

[6] Tomii, N., Tashiro, Y., Tanabe, N., Hirai, C. \& Muraki, K., Train rescheduling algorithm which minimizes passengers' dissatisfaction, Proc. $18^{\text {th }}$ Int. Conf. on Industrial and Engineering Applications of Artificial Intelligence and Expert Systems (IEA/AIE 2005), M. Ali \& F. Esposito eds., LNCS 3533, Springer, pp. 829-838, 2005.

[7] Fujimori, A. \& Tomii, N., A Crew rescheduling algorithm based on combinatorial auction model (in Japanese), Information Technology Letters, 3, LA-006, pp. 15-18, 2004. 